\title{
Fatores associados ao ganho de peso em pacientes com câncer de mama em hormonioterapia
}

\author{
Factors associated with weight gain in breast cancer patients in hormoniotherapy
}

DOI: $10.37111 /$ braspeni.2019344014

Katiúscia Flávia de Lima Silva'

Paulina Nunes da Silva²

Daniela de Araújo Medeiros Dias ${ }^{3}$

\section{Unitermos:}

Neoplasias da mama. Hormônios. Estado nutricional.

\section{Keywords:}

Breast neoplasms. Hormones. Nutritional status.

\section{Endereço para correspondência:}

Daniela de Araújo Medeiros Dias

SQNW 108 Bloco H, apto 311 - Noroeste - Brasilia,

DF, Brasil - CEP 70.686-190

E-mail: danielamedeiros@gmail.com

\section{Submissão}

18 de julho de 2019

\section{Aceito para publicação}

10 de novembro de 2019

\section{RESUMO}

Introdução: A hormonioterapia é um método que consiste no uso de inibidores de hormônios para o tratamento do câncer de mama receptor-positivo, sendo eles receptores de estrogênio e progesterona. Os tipos de hormonioterapia utilizados são os moduladores seletivos do receptor de estrogênio (SERM) e inibidores da aromatase (IA). Destaca-se que a hormonioterapia pode provocar aumento do apetite, retenção hídrica, além de apresentar um ganho de peso progressivo. O objetivo deste estudo é avaliar o estado nutricional de mulheres com câncer de mama em uso de hormonioterapia, em uma clínica particular no Distrito Federal. Método: Foi realizado um estudo transversal, analítico e descritivo, aprovado pelo comitê de ética em pesquisa, durante o período de setembro a outubro de 2018, em uma clínica particular do Distrito Federal, envolvendo pacientes com diagnóstico de câncer de mama em uso de hormonioterapia, do sexo feminino, com idade $\geq 18$ anos. Os parâmetros antropométricos foram avaliados por meio do exame de bioimpedância, circunferência do braço, panturrilha e dobras tricipital e bicipital. Como método de avaliação do estado nutricional e sintomatologia foi aplicada a Avaliação Subjetiva Global Produzida Pelo Paciente (ASG-PPP). Resultados: A amostra foi constituída por 18 mulheres acima de 35 anos, que se encontravam na pré e pós-menopausa em estágio de pós-tratamento da doença, apresentando uma média de IMC de $28 \mathrm{~kg} / \mathrm{m}^{2}$. A maioria das mulheres $(74 \%, \mathrm{n}=$ 13) encontrava-se com sobrepeso e obesidade, sendo que $89 \%$ das participantes apresentaram ganho de peso desde o início do tratamento. Foi também analisada a associação da mudança de peso com a ASG-PPP, sendo que apenas $6 \%$ da população entrevistada se encontravam em risco. Conclusão: Diante dos dados apresentados não se pode afirmar que os resultados obtidos estão unicamente relacionados com o uso dos medicamentos da terapia adjuvante.

\section{ABSTRACT}

Introduction: Hormone therapy is a method consisting of the use of hormone inhibitors for the treatment of receptor-positive breast cancer, estrogen and progesterone receptors. The types of hormone therapy used are selective estrogen receptor modulators (SERM) and aromatase inhibitors (IA). It is noteworthy that hormone therapy can cause increased appetite, water retention, and progressive weight gain. The objective of this staudy is to evaluate the nutritional status of women with breast cancer on hormone therapy at a private clinic in the Federal District. Methods: A cross-sectional, analytical and descriptive study, approved by the Research Ethics Committee, was conducted from September to October 2018, in a private clinic in the Federal District, involving patients diagnosed with breast cancer using hormone therapy, female, aged $\geq 18$ years. Anthropometric parameters were evaluated by bioimpedance, arm circumference, calf, and tricipital and bicipital folds. As a method of assessment of nutritional status and symptomatology was applied the global subjective assessment produced by the patient (ASG-PPP). Results: The sample consisted of 18 women over 35 years old, who were pre- and postmenopausal in the post-treatment stage of the disease, with an average BMl of $28 \mathrm{~kg} / \mathrm{m}^{2}$. Most women $(74 \%, \mathrm{n}=13)$ were overweight and obese, in which $89 \%$ of participants had weight gain since the beginning of treatment. An association of weight change with SGA-PPP was also analyzed, and only $6 \%$ of the interviewed population was at risk. Conclusion: Given the data presented, it cannot be stated that the results obtained are solely related to the use of adjuvant therapy drugs.

1. Graduanda em Nutrição pelo Centro Universitário - UniCEUB, Brasília, DF, Brasil.

2. Nutricionista do Instituto Onco-Vida, Pós-graduação em Nutrição Clínica Enteral e Parenteral - Ganep/SP, e Nutrição Clínica Funcional - Instituto VP/RJ, Brasília, DF, Brasil.

3. Mestre em Nutrição e Saúde pela Universidade Federal de Goiás, professora Adjunta Centro Universitário de Brasília, Brasília, DF, Brasil. 


\section{INTRODUÇÃO}

De acordo com o Instituto Nacional de Câncer (INCA), entre 2018 e 2019, no Brasil, a estimativa é de aproximadamente 600 mil novos casos de câncer; destes, 59.700 são câncer de mama, considerando um risco de 56,33 casos a cada 100 mil mulheres, visto que em 2013, no Brasil, o número de mortes foi de 14.206 mulheres' $^{1}$. Mundialmente, o câncer de mama é o tipo de neoplasia mais incidente na população feminina e caracteriza-se como importante problema de saúde pública².

Atualmente, há diversas terapias para o tratamento do câncer de mama, tais como cirurgia, quimioterapia, radioterapia, hormonioterapia e imunoterapia ${ }^{3}$. A hormonioterapia é um método que consiste no uso de inibidores de hormônios para o tratamento do câncer de mama receptor-positivo ${ }^{4}$, são eles receptores de estrogênio e progesterona $^{3}$. Os tipos de hormonioterapia utilizados são os moduladores seletivos do receptor de estrogênio (SERM) e inibidores da aromatase (IA). Esse tratamento é de grande importância, pois tem impacto significativo na sobrevida dos pacientes e na redução da recorrência da doença ${ }^{5}$.

Segundo Tartari et al. ${ }^{6}$, o tipo de tratamento estabelecido e o próprio tumor comprometem o estado nutricional do paciente. Destaca-se que a hormonioterapia pode provocar aumento do apetite, retenção hídrica, além de apresentar um ganho de peso progressivo. Entretanto, o motivo do ganho de peso pode estar relacionado ao sedentarismo, à qualidade da ingestão alimentar, menopausa ou alteração da taxa metabólica basal. Sabe-se, ainda, que excesso de peso está relacionado com o surgimento de doenças crônicas não-transmissíveis (DCNT), que podem agravar a condição de saúde. Rubin et al. ${ }^{7}$ verificaram, ainda, que a obesidade em mulheres na pós-menopausa é considerada um fator de risco para o desenvolvimento do câncer de mama e está relacionada a uma condição negativa de sobrevida dessas mulheres. As evidências relatam que mulheres com câncer de mama são obesas ou apresentam excesso de peso, que varia entre dois a seis quilos no transcorrer do primeiro ano do diagnóstico².

Ainda são escassos os estudos que avaliaram os fatores de risco para o excesso de peso nessa população e, até o presente momento, não há estudos brasileiros. Dessa forma, se fazem necessários estudos para verificar a causalidade e os fatores associados com o ganho de peso em mulheres em tratamento de hormonioterapia. Diante do exposto, este estudo teve por objetivo avaliar o estado nutricional de mulheres com câncer de mama em uso de hormonioterapia, em uma clínica particular no Distrito Federal.

\section{MÉTODO}

Trata-se de um estudo transversal e descritivo realizado no período de setembro a outubro de 2018 em uma clínica particular oncológica em Brasília, Distrito Federal.

A amostra foi constituída por 18 pacientes com diagnóstico de câncer de mama em tratamento de hormonioterapia, sendo os protocolos prescritos pela equipe médica. A coleta de dados foi realizada em uma instituição particular localizada no Distrito Federal, que se dedica ao tratamento do câncer, priorizando um atendimento multidisciplinar. Foi utilizada uma amostra de conveniência, na qual foram estudadas pacientes do sexo feminino, com idade $\geq 18$ anos, com diagnóstico de câncer de mama em tratamento de hormonioterapia.

Após a seleção das pacientes, foi realizada a coleta de dados demográficos (idade, sexo, estado civil), história clínica (tempo em tratamento e tipo do tratamento), parâmetros antropométricos, como peso atual, peso usual, altura, circunferência do braço, panturrilha, dobras tricipital e bicipital ${ }^{8}$. Foram avaliados a perda ponderal recente, IMC e bioimpedância, utilizando equipamentos de bioimpedância das marcas Inbody 120 e Maltron BF906, estadiômetro compacto da adipômetro e fita métrica, todos da marca Sanny.

Como método de avaliação do estado nutricional, sintomatologia de impacto nutricional e demais características das pacientes, foi utilizada a Avaliação Aubjetiva Global Produzida Pelo Paciente (ASG-PPP). A coleta de dados foi realizada no período de setembro a outubro de 2018.

Foram incluídos pacientes do sexo feminino, com idade $\geq 18$ anos, com diagnóstico de câncer de mama em tratamento de hormonioterapia, independentemente do tipo de tratamento, que concordaram e assinaram o Termo de Consentimento Livre e Esclarecido. Foram excluídas as pacientes impossibilitadas de realizar a antropometria ou responder aos questionários por completo ou aquelas que não compareceram no dia da coleta. Além disso, foram excluídas mulheres com outras doenças associadas que pudessem comprometer o estado nutricional, ascite e/ou edema, em processo infeccioso crônico ou agudo.

Os procedimentos metodológicos do presente trabalho foram preparados dentro dos procedimentos éticos e científicos fundamentais, como disposto na Resolução $N^{\circ} 466$, de 12 de dezembro de 2012, do Conselho Nacional de Saúde do Ministério da Saúde. A presente pesquisa foi aprovada pelo Comitê de ética e pesquisa do Centro Universitário UniCEUB, sob o número 94010818.3.0000.0023.

A análise estatística foi realizada no software Excel da Microsoft ${ }^{\circledR}$ e Statistical Package for Social Sciences (SPSS) versão 17.0. As variáveis categóricas foram descritas em percentuais e números absolutos e as numéricas em média e desvio-padrão ou mediana, valor mínimo e máximo, 
conforme sua distribuição de normalidade (teste de Kolmogorov-Smirnov). Para testar a associação entre IMC, percentual de gordura corporal e circunferência da cintura e variáveis coletadas, foram utilizados os testes de correlação de Pearson e teste $t$ de Student. Adotou-se o nível de significância de 5\%.

\section{RESULTADOS}

A amostra homogênea foi constituída por 18 mulheres com diagnóstico de câncer de mama, com idade média de $49,3 \pm 10,1$ anos (71-35 anos), 67\% eram casadas, $67 \%$ estavam em tratamento do câncer há dois anos e $61 \%$ em tratamento com hormonioterapia há menos de um ano. Não houve diferença estatística das mulheres em relação a idade, tempo diagnóstico e estilo de vida, conforme apresentado na Tabela 1. Ressalta-se que 89\% das mulheres referiram ganho ponderal após a hormonioterapia.

Em relação à avaliação antropométrica, observou-se que $74 \%$ das mulheres apresentavam sobrepeso e obesidade, apresentando uma média de IMC de $28 \mathrm{~kg} / \mathrm{m}^{2}$. Não houve diferença estatística em relação ao estado nutricional.

Em relação à composição corpórea, a média \%CB foi de 108,1 $\pm 13,9$, encontrando-se $6 \%$ em desnutrição moderada, $50 \%$ eutróficas, $33 \%$ com sobrepeso e $11 \%$ apresentaram obesidade; a \%CMB teve como média $106 \pm$

Tabela 1 - Caracterização das mulheres atendidas em uma clínica particular oncológica, 2018.

\begin{tabular}{|c|c|c|c|}
\hline Variáveis & $\mathbf{N}$ & $\%$ & $\mathrm{p}^{*}$ \\
\hline Idade & $\begin{array}{c}49,3 \pm 10,1 \text { anos } \\
\text { (71-35 anos) }\end{array}$ & & \\
\hline Estado civil & & & 0,68 \\
\hline Solteira & 3 & $17 \%$ & \\
\hline Casada & 12 & $67 \%$ & \\
\hline Viúva & 1 & $5 \%$ & \\
\hline Divorciada & 2 & $11 \%$ & \\
\hline $\begin{array}{l}\text { Tempo de tratamento } \\
\text { oncológico }\end{array}$ & $32,1 \pm 18,4$ meses & & 0,74 \\
\hline$<2$ anos & 6 & $33 \%$ & \\
\hline$>2$ anos & 12 & $67 \%$ & \\
\hline Tempo hormonioterapia & $13,7 \pm 3,4$ meses & & 0,88 \\
\hline$<1$ ano & 11 & $61 \%$ & \\
\hline$>1$ ano e $<2$ anos & 5 & $28 \%$ & \\
\hline$>2$ anos & 2 & $11 \%$ & \\
\hline Houve ganho ponderal & & & 0,54 \\
\hline Sim & 16 & $89 \%$ & \\
\hline Não & 2 & $11 \%$ & \\
\hline
\end{tabular}

17,4 , sendo que apenas $5 \%$ apresentaram desnutrição leve, indicando comprometimento da massa muscular. A \%DCT teve a média de $115,3 \pm 23,5$, sendo $44 \%$ classificadas como eutróficas, $11 \%$ com sobrepeso, 39\% com obesidade e apenas $6 \%$ com desnutrição grave (Tabela 2 ).

A média do peso de gordura corporal foi $26,9 \pm 9,6 \mathrm{~kg}$ e de massa magra foi $30,08 \pm 10,5 \mathrm{~kg}$. Foi também analisada relação da mudança de peso com a ASG-PP, no qual apenas $6 \%$ da população entrevistada se encontravam em risco.

Para todas as outras variáveis, não houve correlação ou associação estatística $(p>0,05)$ aos valores de IMC, circunferência da cintura ou percentual de gordura corporal.

Tabela 2 - Avaliação nutricional das mulheres.

\begin{tabular}{|c|c|c|c|}
\hline Variáveis & $\mathbf{N}$ & $\%$ & $\mathbf{p}^{*}$ \\
\hline Peso atual (kg) & $72,3 \pm 12,09^{*}$ & & \\
\hline Peso usual (kg) & $68,5 \pm 10,7^{\star}$ & & \\
\hline Peso ideal (kg) & $58,1 \pm 6,7^{\star}$ & & \\
\hline Altura (m) & $1,60 \pm 0,05^{\star}$ & & \\
\hline$\%$ adequação do peso & $124,1 \pm 14,8^{*}$ & & \\
\hline IMC & $28 \pm 4,2^{*}$ & & \\
\hline$\%$ CB & $108,1 \pm 13,9^{*}$ & & 0,66 \\
\hline Desnutrição moderada & 1 & $6 \%$ & \\
\hline Eutrofia & 9 & $50 \%$ & \\
\hline Sobrepeso & 6 & $33 \%$ & \\
\hline Obesidade & 2 & $11 \%$ & \\
\hline$\% \mathrm{DCT}$ & $115,3 \pm 23,5^{*}$ & & 0,74 \\
\hline Desnutrição grave & 1 & $6 \%$ & \\
\hline Eutrofia & 8 & $44 \%$ & \\
\hline Sobrepeso & 3 & $11 \%$ & \\
\hline Obesidade & 7 & $39 \%$ & \\
\hline$\% \mathrm{CMB}$ & $106 \pm 17,4^{*}$ & $\begin{array}{c}\text { Desnutrição } \\
\text { leve = 5\% }\end{array}$ & \\
\hline$\%$ Gordura corporal & $38,1 \pm 6,7^{*}$ & & 0,088 \\
\hline Muito bom & 1 & $6 \%$ & \\
\hline Bom & 1 & $6 \%$ & \\
\hline Adequado & 4 & $22 \%$ & \\
\hline Alto & 2 & $11 \%$ & \\
\hline Muito alto & 10 & $56 \%$ & \\
\hline Gordura corporal (kg) & $26,9 \pm 9,6^{*}$ & & \\
\hline Massa magra $(\mathrm{kg})$ & $30,08 \pm 10,5^{\star}$ & & 0,09 \\
\hline \multicolumn{4}{|l|}{ ASG-PPP } \\
\hline Com risco & 1 & $6 \%$ & \\
\hline Sem risco & 17 & $94 \%$ & \\
\hline
\end{tabular}




\section{DISCUSSÃO}

Muitos trabalhos demonstraram a ocorrência de ganho de peso durante quimioterapia adjuvante para câncer de mama $^{4,9,10}$. Caan et al. ${ }^{11}$ sugerem pior prognóstico após câncer de mama em pacientes com sobrepeso ou obesidade se comparadas às eutróficas, em estudo duplo-cego com pacientes em uso de tamoxifeno. Sestak et al. ${ }^{12}$ verificaram recorrência do câncer de mama num tempo mediano de 100 meses, sendo que mulheres obesas (IMC> $\left.35 \mathrm{~kg} / \mathrm{m}^{2}\right)$ apresentaram índice de recorrência $46 \%$ maior se comparadas às eutróficas (IMC).

O sobrepeso e a obesidade apresentaram-se de forma expressiva, estando presentes em $74 \%$ da população estudada, no qual $89 \%$ das participantes apresentaram ganho de peso desde o início do tratamento. Corroborando com esse achado, Irwin et al. ${ }^{13}$ também observaram que o ganho de peso e a obesidade são comuns em mulheres com câncer de mama durante o primeiro ano de tratamento, podendo afetar negativamente a sobrevida das mesmas, com recidiva do câncer e aumento do risco de DCNT devido ao excesso de peso. Há necessidade de mais estudos para elucidação dessa causalidade.

Ressalta-se que Arpino et al. ${ }^{14}$ encontraram relação entre aumento de peso e modificações corporais de gordura, acentuando um aumento da circunferência do quadril e da cintura. Seguindo a mesma direção desses resultados, o estudo de Lagares et al. ${ }^{15}$ demonstrou que $87,5 \%$ das pacientes apresentaram grau de obesidade na região abdominal. Dessa forma, faz-se necessário o monitoramento na realização das medidas da circunferência do quadril e cintura, pois são indicadores de risco cardiovascular ${ }^{16}$.

Na presente pesquisa, a média de \%gordura corporal foi $38,1 \pm 6,7$, sendo que $56 \%$ das pacientes foram classificadas com o percentual muito alto, $22 \%$ adequado, $11 \%$ alto, $6 \%$ bom e $6 \%$ muito bom. Estudo realizado com 24 pacientes, em Itaúna, MG, identificou acúmulo de gordura superior, sendo que $91,7 \%$ das mulheres foram classificadas com obesidade e $8,3 \%$ consideradas com sobrepeso, contudo, as variáveis correlacionadas ao percentual de gordura corporal devem-se à ingestão alimentar inadequada, ao maior tempo de menopausa, à idade mais avançada, sendo associadas também a pacientes com hipertensão arterial e diabetes mellitus ${ }^{14}$.

Malinovszky et al. ${ }^{4}$, ao analisarem relatos de pacientes recebendo terapia endócrina adjuvante, encontraram o ganho de peso com maior propensão em mulheres mais jovens na pré-menopausa. Outros sintomas relatados, como náuseas, baixa relação energética e rubores, poderiam estar relacionados ao estágio da doença, ao tipo de terapia adjuvante e, provavelmente, ao status da menopausa das pacientes, concluindo que não se pode relacionar todos os sintomas somente com a terapia endócrina adjuvante, já que não tiveram um grupo controle com idade compatível.

Saquib et al. ${ }^{17}$ observaram que mulheres que fizeram uso do tamoxifeno não tiveram ganho de peso significativo, já $65 \%$ das mulheres que receberam quimioterapia foram mais propensas ao aumento ponderal. Esses autores constataram que, após ganho de peso no início do tratamento, é improvável retornar ao peso anterior ao diagnóstico ${ }^{17}$. Observaram, também, que mulheres com diagnóstico de câncer de mama em idade mais avançada têm menor probabilidade de ganhar peso, porém ressaltam a necessidade de pesquisas com intervenção nutricional e a prática de atividade física, para melhor controle do ganho de peso ${ }^{17}$.

Em estudo de Leite et al. ${ }^{18}$, $87 \%$ das mulheres que fizeram uso do tamoxifeno apresentaram efeitos colaterais, entre eles destaca-se o ganho de peso, em 48,1\% das entrevistadas. Entretanto, o ganho de peso não deve ser unicamente associado ao uso do tamoxifeno, visto que alguns efeitos colaterais possivelmente são pertinentes aos medicamentos utilizados na quimioterapia, uma vez que $63 \%$ das mulheres em uso do tamoxifeno foram submetidas a cirurgia, radioterapia e quimioterapia.

No estudo realizado por Figueiredo et al. ${ }^{19}$, observaram que $80 \%$ das mulheres estavam obesas ou com sobrepeso, de acordo com a classificação do IMC. Durante a pesquisa, a comparação do IMC de pré e pós-tratamento não teve diferença relevante. Observaram que $81,54 \%$ das pacientes em uso do tamoxifeno encontravam-se pré-obesas e obesas. $\mathrm{Na}$ relação do status da menopausa com o IMC pós-tratamento, $64,62 \%$ das mulheres estavam na menopausa, destas $50,77 \%$ eram obesas ou pré-obesas, sendo constatada alta prevalência de mulheres com obesidade e pré-obesidade durante o tratamento do câncer de mama e excesso de peso desde o diagnóstico inicial. Assim sendo, aponta-se a necessidade de estudos mais aprofundados para avaliar o ganho de peso nesse grupo de pacientes.

Uma limitação deste estudo foi a pequena casuística, pois algumas pacientes em tratamento não compareciam à instituição com frequência, devido ao tipo de tratamento a que elas estavam sendo submetidas. Outra limitação é a carência de estudos com resultados semelhantes à presente pesquisa.

\section{CONCLUSÃo}

A maioria das pacientes avaliadas apresentou sobrepeso ou obesidade. Diante dos dados apresentados, não se pode afirmar que os resultados obtidos estão unicamente relacionados com o uso dos medicamentos da terapia adjuvante, pois o ganho ponderal durante o tratamento pode estar relacionado com outros fatores, como ingestão alimentar, sedentarismo e fatores psicossociais. Dessa forma, recomenda-se acompanhamento nutricional para essas mulheres desde o 
início do tratamento do câncer, para garantir bom estado nutricional e melhor qualidade de vida.

Assim, cabe ressaltar a necessidade de estudos para que seja possível concluir a causalidade e os fatores associados com o ganho de peso em mulheres em tratamento de hormonioterapia, além de um maior período de acompanhamento das pacientes.

\section{REFERÊNCIAS}

1. Instituto Nacional do Câncer. Ministério da Saúde. Estimativa 2018: incidência de câncer no Brasil [Internet]. Brasília: Ministério da Saúde; 2014. [acesso em 2018 abril 10]. Disponível em: http://www.inca.gov.br/ estimativa/2018/

2. Poltronieri TS, Tusset C. Impacto do tratamento do câncer sobre o estado nutricional de pacientes oncológicos: atualização da literatura. Rev Bras Ciênc Saúde. 2016;20(4):327-32.

3. Conceição SB. Hormonioterapia no tratamento do câncer de mama: revisão de literatura [trabalho de conclusão de curso]. São Paulo: Hospital do Servidor Público Municipal de São Paulo; 2016.

4. Malinovszky KM, Cameron D, Douglas S, Love C, Leonard T, Dixon JM, et al. Breast cancer patients' experiences on endocrine therapy: monitoring with a checklist for patients on endocrine therapy (C-PET). Breast. 2004;13(5):363-8.

5. Yi M, Hwang E. Pain and menopause symptoms of breast cancer patients with adjuvant hormonal therapy in Korea: secondary analysis. Asia Pac J Oncol Nurs. 2018;5(3):262-9.

6. Tartari RF, Busnello FM, Nunes CHA. Perfil nutricional de pacientes em tratamento quimioterápico em um ambulatório especializado em quimioterapia. Rev Bras Cancerol. 2010;56(1):43-50.

7. Rubin BA, Stein AT, Zelmanowicz AM, Rosa DD. Perfil antropométrico e conhecimento nutricional de mulheres sobreviventes de câncer de mama do sul do Brasil. Rev Bras Cancerol. 2010;56(3):303-9.

8. Lohman TG, Roche AF, Martorell R. Anthropometric standardization reference manual. Champaign: Human Kinetics Books; 1988.
9. Camoriano JK, Loprinzi CL, Ingle JN, Therneau TM, Krook JE, Veeder MH. Weight change in women treated with adjuvant therapy or observed following mastectomy for node-positive breast cancer. J Clin Oncol. 1990;8(8):1327-34.

10. Goodwin PJ, Ennis M, Pritchard KI, McCready D, Koo J, Sidlofsky S, et al. Adjuvant treatment and onset of menopause predict weight gain after breast cancer diagnosis. J Clin Oncol.1999;17(1):120-9.

11. Caan BJ, Kwan ML, Hartzell G, Castillo A, Slattery ML, Sternfeld B, et al. Pre-diagnosis body mass index, post-diagnosis weight change, and prognosis among women with early stage breast cancer. Cancer Causes Control. 2008;19(10):1319-28.

12. Sestak I, Distler W, Forbes JF, Dowsett M, Howell A, Cuzick J. Effect of body mass index on recurrences in tamoxifen and anastrozole treated women: an exploratory analysis from the ATAC trial. J Clin Oncol. 2010;28(21):3411-5.

13. Irwin ML, McTiernan A, Baumgartner RN, Baumgartner KB, Bernstein L, Gilliland FD, et al. Changes in body fat and weight after a breast cancer diagnosis: Influence of demographic, prognostic and lifestyle factors. J Clin Oncol. 2005;23(4):774-82.

14. Arpino G, De Angelis C, Buono G, Colao A, Giuliano M, Malgieri S, et al. Metabolic and anthropometric changes in early breast cancer patients receiving adjuvant therapy. Breast Cancer Res Treat. 2015;154(1):127-32.

15. Lagares EB, Santos KF, Mendes RC, Moreira FA, Anastácio LR. Excesso de peso em mulheres com diagnóstico de câncer de mama em hormonioterapia com tamoxifeno. Rev Bras Cancerol. 2013;59(2):201-10.

16. Guedes DP. Recursos antropométricos para análise da composição corporal. Rev Bras Educ Fís Esp. 2006;20(supl 5):115-9.

17. Saquib N, Flatt SW, Natarajan L, Thomson CA, Bardwell WA, $\mathrm{Caan} \mathrm{B}$, et al. Weight gain and recovery of pre-cancer weight after breast cancer treatments: evidence from the women's healthy eating and living (WHEL) study. Breast Cancer Res Treat. 2007;105(2):177-86.

18. Leite FMC, Bubach S, Amorim MHC, Castro DS, Primo CC. Mulheres com diagnóstico de câncer de mama em tratamento com tamoxifeno: perfil sociodemográfico e clínico. Rev Bras Cancerol. 2011;57(1):15-21.

19. Figueiredo ACDS, Saço LF, Damasceno VO, Ferreira RNF, Ferreira EL. Associação entre variáveis antropométricas e o tratamento para o câncer de mama. ConScientiae Saúde. 2014; 13(1):93-100.

\section{Local de realização do estudo: Centro Universitário - UniCEUB, Brasília, DF, Brasil.}

Conflito de interesse: As autoras declaram não haver.

Artigo proveniente do trabalho de conclusão de curso da graduanda Katiúscia Flávia de Lima Silva.

Foi apresentado como trabalho de destaque no XXIII Congresso Brasileiro de Nutrição Parenteral e Enteral, entre 20 a 23 de outubro de 2019, em Foz do Iguaçu-PR. 